Western Washington University

Western CEDAR

9-1-2010

\title{
Simulations of Luminescent Solar Concentrators: Effects of Polarization and Fluorophore Alignment
}

\author{
Stephen McDowall \\ Western Washington University \\ Brad L. Johnson \\ Western Washington University \\ David L. Patrick \\ Western Washington University, david.patrick@wwu.edu
}

Follow this and additional works at: https://cedar.wwu.edu/chemistry_facpubs

Part of the Chemistry Commons

\section{Recommended Citation}

McDowall, Stephen; Johnson, Brad L.; and Patrick, David L., "Simulations of Luminescent Solar Concentrators: Effects of Polarization and Fluorophore Alignment" (2010). Chemistry Faculty and Staff Publications. 1.

https://cedar.wwu.edu/chemistry_facpubs/1 


\title{
Simulations of luminescent solar concentrators: Effects of polarization and fluorophore alignment
}

\author{
S. McDowall, B. L. Johnson, ${ }^{\text {a) }}$ and D. L. Patrick \\ Advanced Materials Science and Engineering Center, Western Washington University, Bellingham, \\ Washington 98225, USA
}

(Received 9 March 2010; accepted 29 June 2010; published online 3 September 2010)

\begin{abstract}
We model the effects of dye molecule alignment on the collection efficiency of luminescent solar concentrators (LSCs). A Monte Carlo model for photon transport in LSC's is derived and utilized, which incorporates the effects of fluorescent-dye-molecular alignment and the subsequent control over absorption, emission, and propagation properties. We focus on the effects of molecular alignment statistics on photon absorption and subsequent emission, including polarization and propagation direction imparted by dipole direction, to model device light-capture efficiency, defined as the ratio of the amount of light reaching particular slab edges to that incident on a face. We find that modest control of alignment, coupled with reasonable and attainable emission-absorption dipole angles, can produce very large collection efficiencies for a range of device parameters. We note that efficiencies for small values of dye molecule Stoke's shift may be made as large as those for homogeneous (unaligned) systems with large Stoke's shift. (C) 2010 American Institute of Physics. [doi:10.1063/1.3467801]
\end{abstract}

\section{INTRODUCTION}

Luminescent solar concentrators (LSCs) are devices designed to collect incident solar radiation over a large area and concentrate the light onto a small area of matched photovoltaic cells. ${ }^{1-6}$ LSCs are simple devices, consisting of a planar waveguide coated or impregnated with fluorescent dye molecules. Sunlight absorbed by the fluorophore is re-emitted into the waveguide and concentrated at the edges of the collector. The dye molecules absorb solar radiation, and re-emit the light at a longer wavelength, and, depending upon the wavelength separation of the absorption and emission distributions for particular dyes (Stoke's shift), thereby reduce the chance of reabsorption. The slab itself will then act as a waveguide, trapping the collected light and transporting it to the edges of the slab where it can be harvested by band gap-matched solar cells. ${ }^{7,8}$

LSC optical quantum efficiency, defined as the fraction of incident solar photons emitted from the device's edge, depends on several factors: (1) only a certain percentage of incident sunlight is absorbed, depending on the dye concentration and how well its absorption spectrum matches the solar spectrum; (2) only a certain fraction of absorbed light is re-emitted by fluorescence, depending on the dye molecules' fluorescence quantum yield $p_{0}$; and (3) only a certain fraction of this emitted light remains trapped within the waveguide, with the remainder lost out the top or bottom of the slab (so-called escape cone losses). Compounding these inefficiencies is the fact that losses associated with mechanisms (2) and (3) may occur repeatedly, since as photons travel within the slab they may be reabsorbed and re-emitted by other dye molecules more than once before reaching an edge or being lost. There can be other loss mechanisms as well,

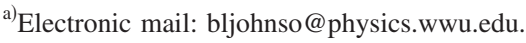

such as scattering from rough surfaces, absorption by the LSC matrix material, etc., but usually these are comparatively small.

As a result of these processes, the overall efficiency is markedly dependent upon the number of reabsorption/reemission events that occur, which is in turn determined by the size of the device, dye concentration, and by the degree of overlap of the dye's absorption and emission spectra. Most efforts aimed at improving LSC performance have focused on the latter property, attempting to minimize selfabsorption through the use of dyes with the largest possible Stoke's shift. However this has proven problematic for several reasons, including the scarcity of stable, highly luminescent dyes having this property. Consequently, despite over 30 years of research, the efficiency of LSCs has remained too low to have any significant practical impact on solar electricity production.

In this work we consider a very different approach to improving LSC efficiency, based on the use of orientationally ordered fluorophores designed to emit light selectively within the plane of the device. There are a variety of ways potentially available for controlling fluorophore orientation in the LSC host matrix, including mechanical alignment by imbedding dyes in a stretched polymer, ${ }^{9}$ the use of liquid crystals via guest-host effects, ${ }^{10-13}$ and if the fluorophore possesses a sufficiently large dipole moment, by poling a polymer/dye composite by electric fields. ${ }^{14}$ Regardless of how orientation is achieved, if the dye molecules are sufficiently well oriented the result is that losses due to mechanism (3) can be virtually eliminated. Likewise, losses due to mechanism (1) can be reduced by increasing dye concentration and/or using a mixture of dyes to cover a larger spectral range. Furthermore, self-absorption in a system of oriented fluorophores can be controlled by engineering the angle between the absorption and emission transition dipoles (see 
below). Finally, since many modern fluorescent dyes have $p_{0} \sim 1$, losses due to mechanism (2) may also be greatly reduced. Thus, the use of orientationally ordered fluorescent waveguide LSCs may offer the potential for major improvements in efficiency.

In order to probe the rather broad parameter space for LSC devices, it is reasonable to model the capture and transport of light in LSCs to guide efforts for constructing and characterizing actual prototypes. Modeling light transport in different media has a long history; models range from transport properties of the atmosphere, ${ }^{15}$ to medical and diagnostic imaging models, ${ }^{16}$ as well as various applications to LSCs. ${ }^{17-20}$ The previous modeling work has focused primarily on calculation of photon yield as a function of geometric constraints, ${ }^{17}$ methods to reduce escape cone losses via inclusion of thin films or other internal reflection mechanisms, ${ }^{8}$ as well as determination of dye concentration and quantum efficiency. ${ }^{20}$ In this paper, we derive a Monte Carlo model and present simulation results for light transport in an optically anisotropic fluorescent medium modeling an oriented fluorophore LSC. The main idea we explore is this: for aligned fluorophores, the absorption probability for a given molecule is governed by the additional constraint of the angle between the polarization of the incident light and the absorption-transition dipole axis. Also, the polarization of light emitted from an aligned molecule depends upon the emission-transition axis; therefore, if the emission and absorption axes for a given molecule are not aligned with each other, subsequent re-absorption may be strongly suppressed by overall alignment of the dye molecules in the device. Furthermore, since the direction of emission from an aligned molecule is determined by the emission-transition axis, alignment of the molecules can also reduce escape cone loss by ensuring that dye emissions are always outside the escape cone for a given material. We find that even modest control of the molecular alignment statistics, combined with relatively modest internal (relative emission-absorption dipole) angles can dramatically enhance the efficiency of LSC devices. In particular, we predict that these modest alignment and angle controls can increase efficiency even for conventional, strongly self-absorbing dyes; if weakly self-absorbing dyes are combined with alignment control, light capture and transport efficiencies significantly exceed those for isotropic systems over a large range of parameter space.

The rest of the paper is organized as follows: in Sec. II, we derive the statistical framework for the Monte Carlo model, including the effects of polarization and dipole alignment on the propagation statistics in the forms most relevant to experimental determination. In Sec. III, we apply the model to a simple slab geometry, investigating the full parameter space of alignment statistics, internal angles, and device length. The results are summarized in Sec. IV.

\section{THEORY}

In this section, we describe in detail how the Monte Carlo simulation of light propagating in a thin-slab of optical material containing a concentration of (aligned) dye mol-

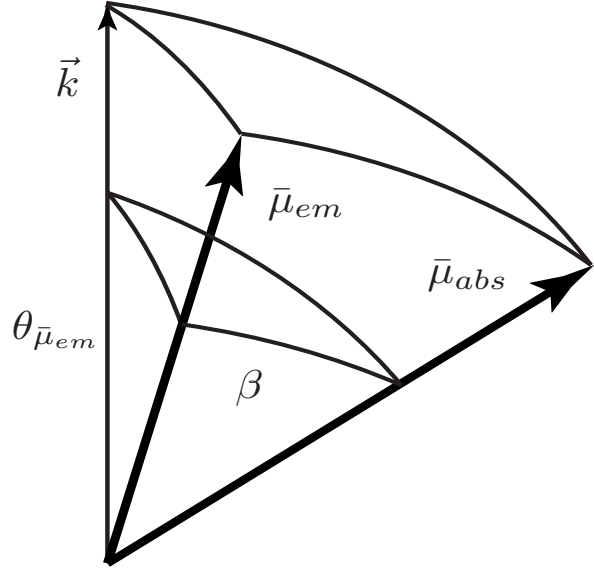

FIG. 1. The geometry of emission and absorption dipoles considered in this paper. Here the emission and absorption dipoles are labeled by $\bar{\mu}_{\mathrm{em}}$ and $\bar{\mu}_{\mathrm{abs}}$, respectively, with $\theta_{\bar{\mu}_{\text {em }}}$ measured between $\vec{k}$ and the emission dipole, and $\beta$ measured between the emission dipole and absorption dipole.

ecules is constructed. The effect of fluorophore alignment statistics is explicitly included, along with the photon polarization.

A photon enters the top of the slab with (unit) velocity vector $\vec{v}$, polarization $\vec{p}$ (which is perpendicular to $\vec{v}$ ), and frequency $\omega$. A typical implementation might be to sample $\vec{v}$ from a prescribed distribution which models the exposure of the top surface of the slab to the illumination source, i.e., direct or diffuse sunlight, and to choose the polarization randomly and uniformly from the circle perpendicular to $\vec{v}$. The frequency $\omega$ is likewise sampled from a distribution modeling the light source such as the AM 1.5 global solar spectrum.

Given the initial $\vec{v}$ and $\omega$, we sample a "transport length" for randomly polarized photons, the statistics for which are derived below. Let us define the transport length of a given photon to be the distance it will travel within the medium before it is absorbed by a dye molecule. The transport length in turn depends on a number of internal parameters. In what follows, the dye molecule transition-dipole for absorption is denoted $\bar{\mu}_{\mathrm{abs}}$, for emission $\bar{\mu}_{\mathrm{em}}$, and the emission dipole makes an angle $\theta_{\bar{\mu}_{\mathrm{em}}}$ with respect to the $z$-axis, while the internal angle between the primary absorption and emission dipoles is labeled $\beta$ (see Fig. 1). In this case, the transport length then depends on the statistics of fluorophore alignment, $\vec{v}, \omega, \beta$, and dye concentration. After propagating one transport length, if the photon is still within the slab, it is absorbed by a second molecule whose emission dipole $\bar{\mu}_{\mathrm{em}}$ is chosen according to the statistics of the alignment. With probability $p_{0}$ the photon is re-emitted; $p_{0}$ is the fluorescent quantum yield of the dye molecule and it is desirable to have $p_{0}$ as close to unity as possible. The direction of emission is chosen at random in a plane perpendicular to $\bar{\mu}_{\mathrm{em}}$, and the photon carries the polarization $\bar{\mu}_{\mathrm{em}}$. The frequency of the new photon is sampled from the probability distribution of the fluorophore's emission spectrum. Now a new transport length is sampled, this time taking into account the new polarization and new frequency. The statistics for this "polarized transport length" are also derived below. The photon then propagates with direction $\vec{v}$; if it encounters the top or 
bottom of the slab, it is either reflected or lost according to the escape cone determined by the difference in refractive indices of the slab to the air. If it encounters an edge, then prescribed boundary conditions determine the action taken. An edge might be mirrored, in which case the photon propagates after reflection; it might be captured by an indexmatched capture device, in which case the photon is captured regardless of its velocity vector; or it might be captured by a capture device which is not index-matched (such as a solar cell), in which case the capture depends on the angle between the velocity vector of the photon and the normal vector to the edge, as well as the ratio of refractive indices. Other boundary conditions might also be chosen. When a photon is successfully captured, its position, velocity vector, and polarization are recorded. A photon which has not escaped from an edge or face of the slab continues to propagate, reflecting as necessary until it is either lost, captured, or exhausts its sampled transport length. In this latter case, the photon is absorbed by a new sampled molecule and the justdescribed process repeats. This is repeated for tens to hundreds of thousands of photons, in order to model the primary transport properties of the device; the low-probability "diffusive" optical transport is thus neglected. We assume that the slab matrix (e.g., polymethylmethacrylate) is optically transparent and does not affect photon polarization, although these effects could be included if desired.

We proceed to describe the derivations of the transport length sampling routines upon which the simulation relies. Denote by $\alpha_{0}=\alpha_{0}(\omega)$ the absorption coefficient for unpolarized light of frequency $\omega$, in units of $\mathrm{cm}^{-1}$; that is to say, if unpolarized light of frequency $\omega$ has initial intensity $I_{0}$, then the expected intensity at distance $x$ into the medium will be

$$
I(x)=I_{0} e^{-\alpha_{0} x}
$$

By unpolarized we mean the photon polarization is (uniformly) randomly distributed about the circle perpendicular to the direction of propagation. Furthermore, (1) is for a medium in which the dye molecules are randomly oriented. Then (1) is equivalent to the probability density function (pdf) with the cumulative density function (cdf) for transport length being

$$
f_{\omega}(x)=\alpha_{0} e^{-\alpha_{0} x} \quad \text { and } \quad F_{\omega}(x)=1-e^{-\alpha_{0} x},
$$

respectively. Transport length can then be sampled by $F_{\omega}^{-1}(y)=\left(-1 / \alpha_{0}\right) \log (y)$, where $y$ is a (uniformly distributed) random number in $[0,1]$.

The absorption coefficient $\alpha_{0}$ takes into account the concentration of the dye by way of the number density $N$ of dye molecules per unit volume. If we then define

$$
\alpha=\alpha(\omega)=\frac{N_{A}}{N} \alpha_{0}(\omega)=\frac{\alpha_{0}}{C},
$$

where $C$ is concentration in units of mol per unit volume, then $\alpha$ has units of unit volume per mole centimeter. Combining this with Eq. (2) we obtain the equivalent representations in terms of $\alpha$

$$
f_{\omega}(x)=C \alpha e^{-\alpha x} \text { and } F_{\omega}(x)=1-e^{-C \alpha x}
$$

and transport length is sampled by

$$
F_{\omega}^{-1}(y)=-1 /(C \alpha) \log (y) .
$$

Equation (3) describes the propagation of unpolarized light in an isotropic (random-dye-orientation) medium. Next, we derive the equivalent parameterizations for unpolarized light in oriented dye molecules and then for polarized light in oriented dye molecules.

When the dye molecules are aligned, and we know some statistics about their alignment, the distributions for transport length change. If $\vec{p}$ is the polarization of a photon which interacts with a dye molecule with absorption dipole $\bar{\mu}_{\text {abs }}$, then the probability of absorption is proportional to $\left(\vec{p} \cdot \bar{\mu}_{\text {abs }}\right)^{2}$; let this constant of proportionality be $k$. If the polarization is considered random, then averaging over $\vec{p}$, we find the probability of absorption conditional on random polarization is $P$ (absorption $\mid$ random $\vec{p}$ ) $=k / 3$. (For brevity, the conditional terms in the probability will be abbreviated by abs and ran, respectively.) Letting $\vec{v}$ be the velocity vector of the photon with random polarization, we compute the probability of absorption conditional on $\vec{v} \cdot \bar{\mu}_{\text {abs }}$ : without loss of generality let $\vec{v}=\vec{k}=(0,0,1)$ and $\bar{\mu}_{\text {abs }}$ $=\left[\sqrt{1-\left(\vec{v} \cdot \bar{\mu}_{\mathrm{abs}}\right)^{2}}, 0, \vec{v} \cdot \bar{\mu}_{\mathrm{abs}}\right]$, and parameterize the polarization vectors $\vec{p}$ by $\vec{p}(\varphi)=(\cos \varphi, \sin \varphi, 0)$. Then

$$
\vec{p}(\varphi) \cdot \bar{\mu}_{\mathrm{abs}}=\cos \varphi \sqrt{1-\left(\vec{v} \cdot \bar{\mu}_{\mathrm{abs}}\right)^{2}},
$$

and so

$$
\begin{aligned}
& P\left(\operatorname{abs} \mid \vec{v} \cdot \bar{\mu}_{\mathrm{abs}}, \operatorname{ran} \vec{p}\right)=\frac{1}{2 \pi} \int_{0}^{2 \pi} k\left[\vec{p}(\varphi) \cdot \bar{\mu}_{\mathrm{abs}}\right]^{2} d \varphi \\
& =\frac{1}{2} k\left[1-\left(\vec{v} \cdot \bar{\mu}_{\mathrm{abs}}\right)^{2}\right] \\
& =\frac{3}{2} P(\operatorname{abs} \mid \operatorname{ran} \vec{p})\left[1-\left(\vec{v} \cdot \bar{\mu}_{\mathrm{abs}}\right)^{2}\right] \\
& =P(\operatorname{abs} \mid \operatorname{ran} \vec{p})\left[1-P_{2}\left(\vec{v} \cdot \bar{\mu}_{\mathrm{abs}}\right)\right],
\end{aligned}
$$

where $P_{2}$ is the second Legendre polynomial. Since Eqs. (3) are derived from $P($ abs $\mid$ ran $\vec{p})$, it follows that the pdf and cdf for transport length for photons with random polarization, conditional on $\vec{v} \cdot \bar{\mu}_{\mathrm{abs}}$, are

$$
\begin{aligned}
f_{\omega}\left(x \mid \vec{v} \cdot \bar{\mu}_{\mathrm{abs}}\right)= & {\left[1-P_{2}\left(\vec{v} \cdot \bar{\mu}_{\mathrm{abs}}\right)\right] \times C \alpha \exp \{[1} \\
& \left.\left.-P_{2}\left(\vec{v} \cdot \bar{\mu}_{\mathrm{abs}}\right)\right] C \alpha x\right\}
\end{aligned}
$$

and

$$
F_{\omega}\left(x \mid \vec{v} \cdot \bar{\mu}_{\text {abs }}\right)=1-\exp \left\{\left[1-P_{2}\left(\vec{v} \cdot \bar{\mu}_{\text {abs }}\right)\right] C \alpha x\right\} .
$$

Recall that in simulating a photon entering the top of the slab, we consider photons with specified velocity vector and random polarization. If the slab has oriented dye molecules, we must calculate the probability of a photon being absorbed conditional on knowing some statistics about $\bar{\mu}_{\text {abs }}$, which depend on the statistics governing the alignment of the dye molecules (see below). Let $g_{\bar{\mu}_{\text {abs }}}$ denote the pdf for $\bar{\mu}_{\text {abs }}$ with respect to the surface measure $d A$ on the sphere $\mathcal{S}^{2}$. We denote this dependence by $\bar{\mu}_{\mathrm{abs}} \sim g_{\bar{\mu}_{\mathrm{abs}}}$. Then, given $\vec{v}$, 


$$
\begin{aligned}
& P\left(\operatorname{abs} \mid \bar{\mu}_{\mathrm{abs}} \sim g_{\bar{\mu}_{\mathrm{abs}}}, \operatorname{ran} \vec{p}\right) \\
& \quad=\int_{\mathcal{S}^{2}} P(\operatorname{abs} \mid \vec{v} \cdot \vec{a}, \operatorname{ran} \vec{p}) g_{\bar{\mu}_{\mathrm{abs}}}(\vec{a}) d A(\vec{a}) \\
& =P(\operatorname{abs} \mid \operatorname{ran} \vec{p}) \int_{\mathcal{S}^{2}}\left[1-P_{2}(\vec{v} \cdot \vec{a})\right] g_{\bar{\mu}_{\mathrm{abs}}}(\vec{a}) d A(\vec{a}) \\
& =P(\operatorname{abs} \mid \operatorname{ran} \vec{p})\left[1-\left\langle P_{2}\left(\vec{v} \cdot \bar{\mu}_{\mathrm{abs}}\right)\right\rangle_{\bar{\mu}_{\mathrm{abs}}}\right],
\end{aligned}
$$

since $g_{\bar{\mu}_{\text {abs }}}$ has unit integral, and where $\langle\cdot\rangle_{\bar{\mu}_{\text {abs }}}$ denotes the average with respect to the density $g_{\bar{\mu}_{\text {abs }}}$. Here, the third line is obtained by substitution of the result Eq. (5). As before, this yields the conditional pdf and cdf for transport length for photons with random polarization, conditional on $\bar{\mu}_{\text {abs }}$ $\sim g_{\bar{\mu}_{\text {abs }}}$,

$$
\begin{aligned}
f_{\omega, \vec{v}}\left(x \mid \bar{\mu}_{\mathrm{abs}} \sim\right. & \left.g_{\bar{\mu}_{\mathrm{abs}}}\right)=\left[1-\left\langle P_{2}\left(\vec{v} \cdot \bar{\mu}_{\mathrm{abs}}\right)\right\rangle_{\bar{\mu}_{\mathrm{abs}}}\right] C \alpha \times \exp \{[1 \\
& \left.\left.-\left\langle P_{2}\left(\vec{v} \cdot \bar{\mu}_{\mathrm{abs}}\right)\right\rangle_{g_{\bar{\mu}_{\mathrm{abs}}}}\right] C \alpha x\right\},
\end{aligned}
$$

and

$$
\begin{aligned}
F_{\omega, \vec{v}}\left(x \mid \bar{\mu}_{\mathrm{abs}} \sim\right. & \left.g_{\bar{\mu}_{\mathrm{abs}}}\right)=1-\exp \{[1 \\
& \left.\left.-\left\langle P_{2}\left(\vec{v} \cdot \bar{\mu}_{\mathrm{abs}}\right)\right\rangle_{g_{\bar{\mu}_{\mathrm{abs}}}}\right] C \alpha x\right\} .
\end{aligned}
$$

At this point, we can make an important observation: If we define an effective concentration $C_{\mathrm{eff}}(\omega, \vec{v})=C[1$ $\left.-\left\langle P_{2}\left(\vec{v} \cdot \bar{\mu}_{\text {abs }}\right)\right\rangle_{g_{\bar{\mu}_{\mathrm{abs}}}}\right]$ then for photons with velocity $\vec{v}$ with random polarization entering a medium with aligned dye molecules, we can sample the transport length via

$$
F_{\omega, \vec{v}}^{-1}\left(\cdot \mid \bar{\mu}_{\mathrm{abs}} \sim g_{\bar{\mu}_{\mathrm{abs}}}\right)(y)=\frac{-1}{C_{\mathrm{eff}}(\omega, \vec{v}) \alpha(\omega)} \log (y),
$$

with $y$ chosen randomly from [0,1]. This has the familiar form, analogous to the unpolarized case given by Eq. (4).

In order to apply the derived sampling of transport length for a photon with given velocity $\vec{v}$, we must compute $\left\langle P_{2}(\vec{v} \cdot \vec{a})\right\rangle_{g_{\bar{\mu}_{\mathrm{abs}}}}$. We begin by describing the model for alignment of the dye molecules. We use a Euclidean coordinate system with orthonormal basis $\{\vec{i}, \vec{j}, \vec{k}\}$. We describe dye molecule alignment in terms of the statistics of the angle $\theta_{\bar{\mu}_{\mathrm{em}}}$ between $\bar{\mu}_{\mathrm{em}}$ and $\vec{k}$-see Fig. 1. In an actual experiment, where dye orientation is measured using polarized fluorescence spectroscopy, one usually determines $P_{2}\left(\cos \theta_{\bar{\mu}_{\mathrm{em}}}\right)$ $=P_{2}\left(\bar{\mu}_{\mathrm{em}} \cdot \vec{k}\right)$, which is typically expressed as an order parameter $S_{\bar{\mu}_{\mathrm{em}}}=\left\langle P_{2}\left(\cos \theta_{\bar{\mu}_{\mathrm{em}}}\right)\right\rangle^{21}$ From knowledge of $S_{\bar{\mu}_{\mathrm{em}}}$ we approximate the pdf for $\theta_{\bar{\mu}_{\text {em }}}$ as a function of the polar angle $\theta$ via the principle of maximum entropy

$$
\theta_{\bar{\mu}_{\mathrm{em}}} \sim h_{\bar{\mu}_{\mathrm{em}}}(\theta):=\frac{\exp \left[c_{2} P_{2}(\cos \theta)\right]}{\int_{0}^{\pi} \exp \left[c_{2} P_{2}\left(\cos \theta^{\prime}\right)\right] \sin \theta^{\prime} d \theta^{\prime}},
$$

where $c_{2}$ satisfies

$$
\begin{aligned}
S_{\bar{\mu}_{\mathrm{em}}} & =\left\langle P_{2}\left(\cos \theta_{\bar{\mu}_{\mathrm{em}}}\right)\right\rangle \\
& =\frac{\int_{0}^{\pi} P_{2}(\cos \theta) \exp \left[c_{2} P_{2}(\cos \theta)\right] \sin \theta d \theta}{\int_{0}^{\pi} \exp \left[c_{2} P_{2}(\cos \theta)\right] \sin \theta d \theta} .
\end{aligned}
$$

This gives the pdf $g_{\bar{\mu}_{\mathrm{em}}}(\bar{\mu}) d A(\bar{\mu})=\frac{1}{2 \pi} h_{\bar{\mu}_{\mathrm{em}}}(\theta) \sin \theta d \theta d \varphi$ for $\bar{\mu}_{\mathrm{em}}$ in spherical coordinates; we can also express this in Euclidean coordinates as $g_{\bar{\mu}_{\mathrm{em}}}(\bar{\mu})=\frac{1}{2 \pi d} \exp \left[c_{2} P_{2}(\bar{\mu} \cdot \vec{k})\right]$, where $d$ is the normalizing denominator in Eq. (9).

As noted above, the angle between the primary emission dipole and the primary absorption dipole is denoted by $\beta$ (see Fig. 1). To compute $g_{\bar{\mu}_{\text {abs }}}\left(\bar{\mu}_{\text {abs }}\right)$, we integrate $g_{\bar{\mu}_{\text {em }}}$ over the circle in $\mathcal{S}^{2}$ of the emission vectors $\bar{\mu}_{\mathrm{em}}$ which make angle $\beta$ with the absorption vector $\bar{\mu}_{\text {abs }}$. Since $g_{\bar{\mu}_{\text {em }}}$ is independent of $\varphi_{\bar{\mu}_{\mathrm{em}}}$, the polar angle of $\bar{\mu}_{\mathrm{em}}$, so too will be $g_{\bar{\mu}_{\text {abs }}}$ and so without loss of generality we may assume that $\bar{\mu}_{\text {abs }}$ $=\left(\sin \theta_{\bar{\mu}_{\mathrm{abs}}}, 0, \cos \theta_{\bar{\mu}_{\mathrm{abs}}}\right)$. In spherical coordinates,

$$
\begin{aligned}
& \theta_{\bar{\mu}_{\mathrm{abs}}} \sim h_{\bar{\mu}_{\mathrm{abs}}}(\theta), \\
& g_{\bar{\mu}_{\mathrm{abs}}}(\bar{\mu}) d A(\bar{\mu})=\frac{1}{2 \pi} h_{\bar{\mu}_{\mathrm{abs}}}(\theta) \sin \theta d \theta d \varphi, \\
& h_{\bar{\mu}_{\mathrm{abs}}}(\theta)=\frac{h_{0}(\theta)}{\int_{0}^{\pi} h_{0}\left(\theta^{\prime}\right) \sin \theta^{\prime} d \theta^{\prime}}, \\
& \bar{\mu}_{\mathrm{em}}(\alpha)=\sin \beta\left(\cos \alpha \bar{\mu}_{\mathrm{abs}}^{\perp} \times \bar{\mu}_{\mathrm{abs}}+\sin \alpha \bar{\mu}_{\mathrm{abs}}^{\perp}\right) \\
& +\cos \beta \bar{\mu}_{\mathrm{abs}},
\end{aligned}
$$

for $h_{0}(\theta)$ which we proceed to compute. Setting $\bar{\mu}_{\mathrm{abs}}^{\perp}$ $=(0,1,0)$, the circle of $\bar{\mu}_{\text {em }}$ making an angle $\beta$ with $\bar{\mu}_{\text {abs }}$ can

for $0 \leq \alpha \leq 2 \pi$, from which we obtain

$$
\bar{\mu}_{\mathrm{em}}(\alpha) \cdot \vec{k}=-\sin \beta \cos \alpha \sin \theta_{\bar{\mu}_{\mathrm{abs}}}+\cos \beta \cos \theta_{\bar{\mu}_{\mathrm{abs}}} .
$$

Thus, using the Euclidean expression for $g_{\bar{\mu}_{\text {abs }}}$,

$$
h_{0}\left(\theta_{\bar{\mu}_{\mathrm{abs}}}\right)=\int_{0}^{2 \pi} \exp \left[c_{2} P_{2}\left(\bar{\mu}_{\mathrm{em}} \cdot \vec{k}\right)\right] d \alpha,
$$

with

$$
\bar{\mu}_{\mathrm{em}} \cdot \vec{k}=\cos \beta \cos \theta_{\bar{\mu}_{\mathrm{abs}}}-\sin \beta \cos \alpha \sin \theta_{\bar{\mu}_{\mathrm{abs}}} .
$$

Having computed $g_{\bar{\mu}_{\text {abs }}}$, for given $\vec{v}$, we now compute $\left\langle P_{2}(\vec{v} \cdot \bar{\mu})\right\rangle_{\bar{\mu}_{\text {abs }}}$ by integrating $P_{2}(\vec{v} \cdot \bar{\mu})$ against $g_{\bar{\mu}_{\mathrm{abs}}}(\bar{\mu}) d A(\bar{\mu})$ over $\mathcal{S}^{2}$. Without loss of generality, we may let $\vec{v}$ $=\left(\sin \theta_{0}, 0, \cos \theta_{0}\right)$. Then,

$$
\begin{aligned}
& \left\langle P_{2}(\vec{v} \cdot \bar{\mu})\right\rangle_{g_{\bar{\mu}_{\mathrm{abs}}}}=\frac{1}{16} \int_{0}^{\pi}\left[1+3 \cos \left(2 \theta_{0}\right)\right] \\
& \times[1+3 \cos (2 \theta)] h_{\bar{\mu}_{\mathrm{abs}}} \sin \theta d \theta,
\end{aligned}
$$

where the functional dependence $h_{\bar{\mu}_{\mathrm{abs}}}(\theta)$ has been suppressed for brevity.

In summary, from knowledge of only the statistic $\left\langle P_{2}\left(\bar{\mu}_{\mathrm{em}} \cdot \vec{k}\right)\right\rangle$ for the alignment of the dye molecules, we have derived the expression for the effective concentration $C_{\text {eff }}(\omega, \vec{v})$ and hence the mechanism for sampling transport 
length for photons at frequency $\omega$, velocity vector $\vec{v}$, and with random polarization. This enables the first step in the Monte Carlo simulation of photon transport within a slab with oriented dye molecules, as photons enter the slab.

Beyond this first step, the photons can no longer be considered to have random polarization since they have been emitted from oriented dye molecules. Indeed, the polarization vectors carry the statistics of the emission dipoles $\bar{\mu}_{\mathrm{em}}$. We must now derive a sampling scheme for transport length for such a photon. We have,

$$
P(\operatorname{abs} \mid \vec{p})=\int_{\mathcal{S}^{2}} P(\operatorname{abs} \mid \vec{p}, \bar{\mu}) g_{\bar{\mu}_{\mathrm{abs}}}(\bar{\mu}) d A(\bar{\mu})
$$

and $P(\operatorname{abs} \mid \vec{p}, \bar{\mu})=k(\vec{p} \cdot \bar{\mu})^{2}$. As remarked earlier, $k$ $=3 P(\operatorname{abs} \mid \operatorname{ran} \vec{p})$. Let the polarization $\vec{p}$ have spherical coordinates $\left(\theta_{\vec{p}}, \varphi_{\vec{p}}\right)$. Then

$$
\begin{aligned}
P(\operatorname{abs} \mid \vec{p}) & =\int_{\mathcal{S}^{2}} k(\vec{p} \cdot \bar{\mu})^{2} g_{\bar{\mu}_{\mathrm{abs}}}(\bar{\mu}) d A(\bar{\mu}) \\
& =P(\operatorname{abs} \mid \operatorname{ran} \vec{p}) p_{\omega}\left(\theta_{\vec{p}}\right),
\end{aligned}
$$

with

$$
\begin{aligned}
& p_{\omega}\left(\theta_{\vec{p}}\right)= 3 \int_{0}^{\pi} \int_{0}^{2 \pi}\left[\vec{p}\left(\theta_{\vec{p}}, \varphi_{\vec{p}}\right) \cdot \bar{\mu}(\theta, \varphi)\right]^{2} \\
& \times \frac{1}{2 \pi} h_{\bar{\mu}_{\mathrm{abs}}} \sin (\theta) d \theta d \varphi \\
&= \frac{3}{8} \int_{0}^{\pi}\left[3+\cos 2 \theta_{\vec{p}}+\cos 2 \theta\right. \\
&\left.\quad \times\left(1+3 \cos 2 \theta_{\vec{p}}\right)\right] h_{\bar{\mu}_{\mathrm{abs}}} \sin \theta d \theta .
\end{aligned}
$$

Once again, this yields the conditional pdf and cdf for transport length, conditional on $\vec{p}$ and $\bar{\mu}_{\text {abs }} \sim g_{\bar{\mu}_{\text {abs' }}}$,

$$
\begin{aligned}
& f_{\omega}\left(x \mid \vec{p}, \bar{\mu}_{\mathrm{abs}} \sim g_{\bar{\mu}_{\mathrm{abs}}}\right)=p_{\omega}\left(\theta_{\vec{p}}\right) C \alpha \exp \left[p_{\omega}\left(\theta_{\vec{p}}\right) C \alpha x\right], \\
& F_{\omega}\left(x \mid \vec{p}, \bar{\mu}_{\mathrm{abs}} \sim g_{\bar{\mu}_{\mathrm{abs}}}\right)=1-\exp \left[p_{\omega}\left(\theta_{\vec{p}}\right) C \alpha x\right],
\end{aligned}
$$

and we sample transport length via

$$
F_{\omega}^{-1}\left(\cdot \mid \vec{p}, \bar{\mu}_{\mathrm{abs}} \sim g_{\bar{\mu}_{\mathrm{abs}}}\right)(y)=\frac{-1}{p_{\omega}\left(\theta_{\vec{p}}\right) C \alpha(\omega)} \log (y),
$$

with $y$ chosen randomly from [0,1]. In Sec. III, we will apply the polarization and alignment formalism to a specific geometry, in order to demonstrate the possible efficiency gains attained for ranges of alignment statistics.

\section{RESULTS}

In this section, we will apply the model described above to a numerical simulation. Specifically, we investigate the performance of a particular solar concentrator geometry by running Monte Carlo simulations for a variety of parameter configurations. Our principal goal in the present article is to demonstrate the dependence of performance, as a function of side-length of the concentrator, on the degree of alignment of the dye molecules, and also on the angle $\beta$ between the emis-

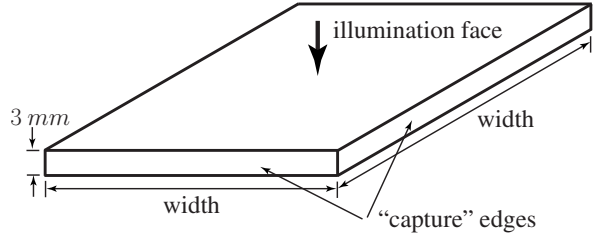

FIG. 2. The concentrator geometry considered for the numerical simulations. The slab is a square, illuminated on the top face, and is $3 \mathrm{~mm}$ thick.

sion and absorption dipoles of the dye molecules. We also wish to compare this with performance for slabs with unaligned molecules. We quantify performance by the percent of photons entering the top face of the slab which are captured at an edge-the so-called optical quantum efficiency. In the simulations reported here we illuminate the slab with monochromatic photons striking the top face at its center and with direction perpendicular to the face. The slab is taken to be a square of dimensions from $2 \times 2 \mathrm{~cm}$ through 64 $\times 64 \mathrm{~cm}$, and $0.3 \mathrm{~cm}$ thick. The simulation geometry is sketched in Fig. 2.

One of the principal characteristics of dye molecules that erodes the efficiency of solar collectors/concentrators is the ability of a fluorophore to absorb its own light (selfabsorption). Self-absorption is most conveniently characterized by the unit-less parameter ${ }^{22} S_{\mathrm{abs}}=A_{\mathrm{pa}} / A_{\mathrm{pe}}$, where $A_{\mathrm{pa}}$ is the absorbance at the fluorophore peak absorption wavelength and $A_{\mathrm{pe}}$ is the absorbance at the peak emission wavelength. The range of $S_{\mathrm{abs}}$ for typical dyes is 2-20. In general, a large $S_{\text {abs }}$ reduces self-absorption losses. ${ }^{22}$ We shall show by our simulations that for the moderate value of $S_{\mathrm{abs}}=10$ we can obtain superior performance by utilizing alignment; in all the simulations where a certain degree of alignment is employed, we will take $S_{\text {abs }}=10$.

Performance also depends significantly on the concentration of the dye within the slab, for the following reasons: if the concentration is low, the probability of an incident photon being absorbed before passing through the $0.3 \mathrm{~cm}$ thick slab is low and the resulting performance is very poor. On the other hand, photons which are absorbed and re-emitted into the slab travel long distances before being reabsorbed, and so have a greater probability of being captured at an edge. Conversely, a high concentration increases the proportion of photons which experience an initial absorption-reemission event and so are transported within the slab but they experience a higher number of absorption-re-emission events before reaching an edge, and so are more likely to be lost either to nonradiative relaxation mechanisms or out the escape cones of the top and bottom faces.

When a photon interacts with a dye molecule, the probability of absorption depends (among other things) on the energy of the photon relative to the absorption spectrum of the dye. To standardize results, we fix an absorption profile to be Gaussian with an arbitrarily chosen mean $\mu_{\mathrm{ab}}=4$ and variance $\sigma_{\mathrm{ab}}=0.5$. All incoming photons entering the slab on the top face are given energy equal to $\mu_{\mathrm{ab}}$. The photon is then re-emitted with a probability determined by the fluorescence quantum yield of the dye, which throughout is taken to be $p_{o}=0.95$; at every absorption event, there is a $5 \%$ chance of 


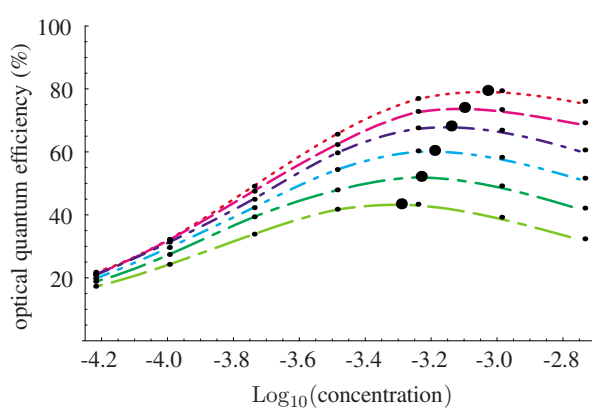

FIG. 3. (Color online) An illustration of the calculation for optimal concentration for each geometry, so that the effects of alignment may be isolated. Here $S_{\mathrm{abs}}=10, S_{\bar{\mu}_{\mathrm{em}}}=0.8$, and $\beta=30$, for side widths given by $2^{n}, 1 \leq n \leq 6$ from top curve to bottom, respectively.

the photon being lost to radiationless transitions. If the photon is re-emitted, then its energy is sampled according to the fluorescence emission spectrum of the dye. This distribution is again taken to be a Gaussian, of the same variance as the absorption profile but with mean shifted so as to result in the dye having a particular value of the parameter $S_{\text {abs }}$. Precisely, $\mu_{\mathrm{em}}=\mu_{\mathrm{ab}}-\sqrt{2 \sigma_{\mathrm{ab}}} \sqrt{\ln \left(S_{\mathrm{abs}}\right)}$.

As described in Sec. II above, we quantify alignment by the (experimental) order parameter $S_{\bar{\mu}_{\mathrm{em}}}=\left\langle P_{2}\left(\cos \theta_{\bar{\mu}_{\mathrm{em}}}\right)\right\rangle$ $=\left\langle P_{2}\left(\bar{\mu}_{\mathrm{em}} \cdot \vec{k}\right)\right\rangle$, the average taken with respect to the measure $\sin \theta d \theta$, and where $P_{2}$ is the second Legendre polynomial. When $S_{\bar{\mu}_{\mathrm{em}}}=0$, the alignment of the molecules is random (we refer to the dye as unaligned).

In order to remove concentration as a factor, we perform initial simulations to determine an optimal concentration for a given configuration. More precisely, given a choice of parameters $\beta$ and $S_{\bar{\mu}_{\text {em }}}$, for each side-length we run simulations with dye concentrations $10^{\alpha}$ for $\alpha=-4.25,-4,-3.75, \ldots$, $-3,-2.75$. From the resulting data we construct a cubic interpolation (performance as a function of concentration) and determine the location of the maximum, which produces the optimal concentration for each slab size. A sample of the procedure is illustrated in Fig. 3. It is this optimal concentration that we use in the subsequent simulations and so we can conclude that any observed improvement in performance is due solely to $\beta$ and $S_{\bar{\mu}_{\mathrm{em}}}$, and not due to concentration choice.

For a given choice of $\beta$ and $S_{\bar{\mu}_{\mathrm{em}}}$ we now run the Monte Carlo simulation for a large number of photons. Certain probability distributions need to be computed before the simulation can be executed. We numerically solve Eq. (10) for $c_{2}$ and thus, by Eq. (9), define the pdf $h_{\bar{\mu}_{\mathrm{em}}}$ for $\theta_{\bar{\mu}_{\mathrm{em}}}$, the angle between the emission dipole and the vector $\vec{k}$. From this we compute a numerical approximation to the inverse $H_{\bar{\mu}_{\mathrm{em}}}^{-1}$ of the corresponding cdf; we are thus able to sample $\theta_{\bar{\mu}_{\mathrm{em}}}$ via $H_{\bar{\mu}_{\mathrm{em}}}^{-1}(y)$ with $y$ chosen randomly and uniformly from $[0,1]$. This is used to randomly sample a dye molecule as follows: having sampled $\theta_{\bar{\mu}_{\mathrm{em}}}$ we randomly choose a vector from the circle of vectors in the unit sphere which make angle $\theta_{\bar{\mu}_{\text {em }}}$ with $\vec{k}$. This defines a sampled emission dipole $\bar{\mu}_{\text {em }}$. Next, having chosen $\bar{\mu}_{\text {em }}$, we randomly choose a vector from the circle of vectors in the unit sphere which make an angle $\beta$ with $\bar{\mu}_{\mathrm{em}}$. This defines the sampled absorption dipole $\bar{\mu}_{\mathrm{ab}}$.

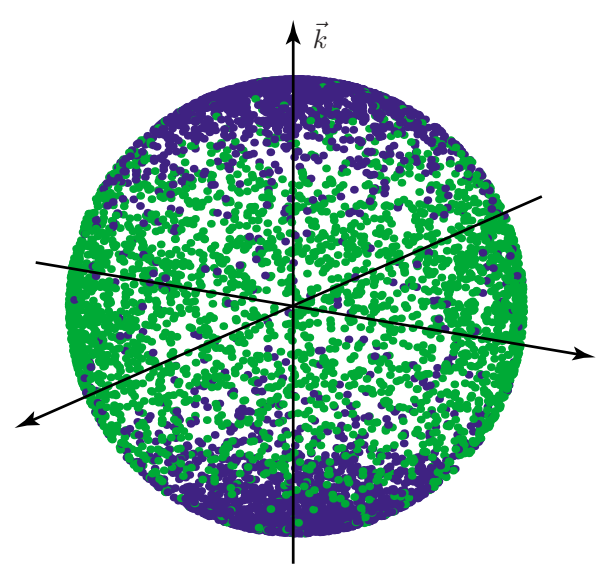

FIG. 4. (Color online) A sampled distribution of emission dipoles (dark circle) and corresponding photon distribution (light circle), for $S_{\bar{\mu}_{\text {abs }}}=0.6$.

The numerical strategy is constructed as follows; we see that we must compute $h_{0}(\theta)$ from Eq. (11) which we approximate numerically, and which in turn defines $h_{\bar{\mu}_{\text {abs }}}$. We then, again numerically, compute the function $\vec{v} \mapsto\left\langle P_{2}(\vec{v} \cdot \bar{\mu})\right\rangle_{\bar{\mu}_{\text {abs }}}$ according to Eq. (12). This completes the ingredients to sample transport length for the unpolarized incoming photons entering at the top of the slab. Finally, we compute $p_{\omega}\left(\theta_{\vec{p}}\right)$ [see Eq. (13)] which facilitates sampling transport length for polarized photons by way of Eq. (14). We remark that, while the numerical approximations of the probability distributions described above might be somewhat timeconsuming, they need only be done once for a given choice of parameters $\beta$ and $S_{\bar{\mu}_{\text {em }}}$. Furthermore, for this reason, they can be computed to a very high degree of accuracy at no cost to the total run-time of the simulation.

We run the Monte Carlo simulation on slabs of lateral dimensions $2^{n} \times 2^{n}$ for $1 \leq n \leq 6$. We consider alignment statistics of $S_{\bar{\mu}_{\mathrm{abs}}}=0.6$ (moderately aligned) and $S_{\bar{\mu}_{\mathrm{abs}}}=0.8$ (wellaligned). We note that this range of order parameters has been experimentally achieved for hundreds of fluorescent dyes using liquid crystal alignment media and other methods. ${ }^{13,23}$ In order to clarify the effect of the alignment statistics, we show the distributions of emission dipoles, as well as the corresponding distributions for the subsequent photon emission, in Figs. 4 and 5 for values of $S_{\bar{\mu}_{\text {abs }}}=0.6$ and



FIG. 5. (Color online) A sampled distribution of emission dipoles (dark circle) and corresponding photon distribution (light circle), for $S_{\bar{\mu}_{\text {abs }}}=0.8$. 


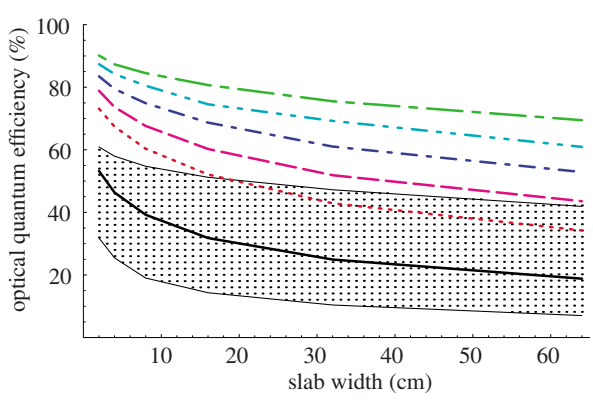

FIG. 6. (Color online) The optical quantum efficiency vs slab width for fixed $S_{\bar{\mu}_{\mathrm{abs}}}=0.8$. The curves are for $\beta=15^{\circ}, 30^{\circ}, 45^{\circ}, 60^{\circ}$, and $75^{\circ}$ for the dotted through the dashed-dotted curves. The shaded region represents simulation results for unaligned dyes, with boundaries given by the range of values for $S_{\mathrm{abs}}$ and $p_{0}$ from the literature: bottom $S_{\mathrm{abs}}=5.4, p_{0}=0.78$ (Ref. 24); top $S_{\text {abs }}=220, p_{0}=0.9$ (Ref. 25). The solid curve is for $S_{\text {abs }}=10, p_{0}$ $=0.95$.

$S_{\bar{\mu}_{\mathrm{abs}}}=0.8$, respectively. Here the distribution of blue (dark) dots represents the location of the emission dipole intersecting the sphere, while the distribution of green (light) dots is that of the corresponding emitted photon distribution. The figures depict the effect of statistical variations associated with the different values of $S_{\bar{\mu}_{\text {abs }}}$.

In order to illustrate and quantify the performance of solar concentrators as a function of the alignment statistics, we show simulation results in two categories: fixing the alignment statistics and examining the effects of the internal angle $\beta$, and fixing the internal angle and varying the alignment statistics. In all cases, we plot the optical quantum efficiency of the slab as a function of the slab width in centimeters, with a fixed value of the self-absorption parameter $S_{\text {abs }}$ and fluorescent quantum yield $p_{0}$. Also, in all cases, simulations of aligned systems are compared directly to similar simulations for randomly-aligned systems under different conditions. The unaligned systems have a variety of self-absorption parameters $S_{\text {abs }}$ and fluorescent quantum yields, representing a range of values reported in the literature.

In Fig. 6, we show the optical quantum efficiency as a function of slab width, for a fixed value of alignment statistics $S_{\bar{\mu}_{\mathrm{abs}}}=0.8$. The shaded region in the figure depicts the range of performance produced by our simulations using values of $S_{\mathrm{abs}}$ and $p_{0}$ reported in the literature, with $S_{\bar{\mu}_{\mathrm{abs}}}=0$ (unaligned dyes). The boundaries of the shaded region are obtained via simulations with $S_{\text {abs }}=5.4$ and $p_{0}=0.78$ (bottom curve) (Ref. 24) and $S_{\text {abs }}=220$ and $p_{0}=0.9$ for the top curve, ${ }^{25}$ respectively. All other reported values result in curves which lie within the shaded region. The solid line curve is for $S_{\text {abs }}=10$ for the unaligned case, $S_{\bar{\mu}_{\text {abs }}}=0$, with $p_{0}=0.95$. The other curves are given as follows: $S_{\mathrm{abs}}=10$ and $\beta=15^{\circ}, 30^{\circ}, 45^{\circ}, 60^{\circ}$, and $75^{\circ}$ for the lowest (dotted) curve through the highest (dashed-dotted) curves, respectively. We note a significant increase in the slab efficiency with internal angle; the case $\beta=75$ remains nearly $80 \%$ efficient for slabs up to $60 \mathrm{~cm}$ in width.

Figure 7 shows the optical quantum efficiency versus width for a set of systems with fixed internal angle $\beta=30^{\circ}$ and $p_{0}=0.95$, for a series of different $S_{\text {abs }}$ and $S_{\bar{\mu}_{\text {abs }}}$. For the lower two curves (dotted and dashed), $S_{\text {abs }}=10$ and $S_{\bar{\mu}_{\text {abs }}}$

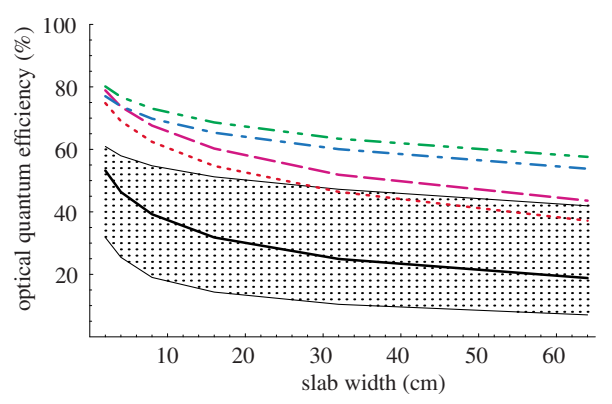

FIG. 7. (Color online) The optical quantum efficiency vs slab width for fixed $\beta=30^{\circ}$, with $S_{\bar{\mu}_{\text {abs }}}=0.6$ and 0.8 , for each of the two pairs of broken curves; for the lower pair (dotted and dashed) $S_{\text {abs }}=10$, while for the upper pair $S_{\text {abs }}=80$.

$=0.6$ and 0.8 , respectively, with $p_{0}=0.95$. For the upper two curves (dashed-dotted and dashed-dotted-dotted), $S_{\mathrm{abs}}=80$ and $S_{\bar{\mu}_{\mathrm{abs}}}=0.6$ and 0.8 , respectively and $p_{0}=0.9$; these values make for a more direct comparison with the literature values for the unaligned systems depicted in the shaded region - the shaded region is once again the simulation data for a range of unaligned systems, as in Fig. 6. We note that even with the modest value of $\beta=30^{\circ}$, the performance may be significantly enhanced via alignment control.

Lastly, Fig. 8 is similar to that of Fig. 7, except that here $\beta=75^{\circ}$. In this figure, $S_{\mathrm{abs}}=10$ and $p_{0}=0.95$ for the broken curves, with $S_{\bar{\mu}_{\text {abs }}}=0.6,0.7,0.8$, nad, 0.9 , for the lowest (dotted) through the highest (dashed-dotted-dotted) curves, respectively. Note that the combination of the large angle $\beta$ and alignment control contributes to a significant performance enhancement over unaligned systems.

The efficiency gains depicted in Figs. 7 and 8 indicate the overall effect of fluorophore alignment; it is interesting to note, however, that any mechanism that reduces escape cone losses-and thereby traps photons in the slab for longer trajectories-must then increase the number of absorption and emission events. In Fig. 9, we show the average number of photon absorption events as a function of the order parameter $S_{\bar{\mu}_{\text {abs }}}$ for a simulation with a fixed side-length of 32 $\times 32 \mathrm{~cm}, \beta=30$, and $S_{\mathrm{abs}}=10$. The three curves correspond to absorption events for photons captured through the edges (solid line), absorbed and lost due to fluorescent quantum efficiency (dashed line), and escaping out of the top and bottom faces (dashed-dotted curve). We note that indeed the captured photons (as well as the number of photons lost to efficiency) undergo more absorptions with increasing align-

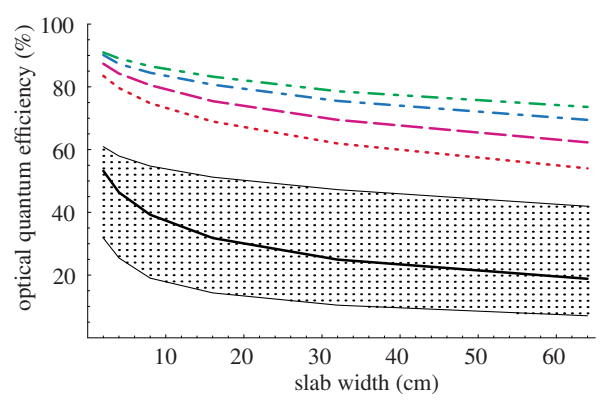

FIG. 8. (Color online) The optical quantum efficiency vs slab width for fixed $\beta=75^{\circ}$, with $S_{\bar{\mu}_{\text {abs }}}=0.6,0.7,0.8$, and 0.9 , for the dotted through the dashed-dotted-dotted curves. 


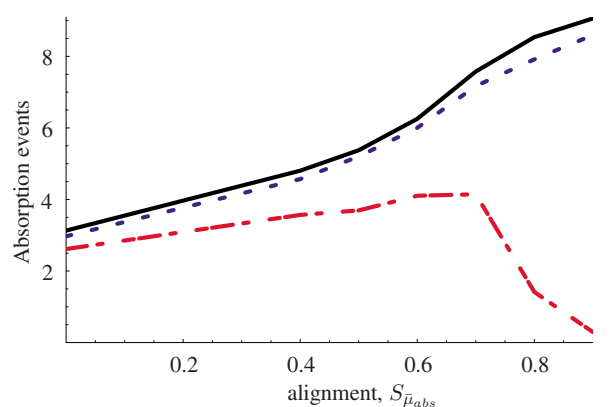

FIG. 9. (Color online) The average number of absorption and emission events for photons that are captured (solid line), lost via fluorescent quantum efficiency (dashed line), and escape (dashed-dotted line).

ment, due to the reduction in escape cone losses; what is evident from the figure is that the reduction in escape cone losses is much more significant than the loss due to increased self-absorption. To further quantify the relationship, Fig. 10 depicts the percentage of photons captured or lost as a function of $S_{\bar{\mu}_{\text {abs }}}$ for the same parameters as Fig. 9. As before, the solid curve is for captured photons, the dashed curve for photons lost to fluorescent quantum efficiency and the dashed-dotted curve depicts escape cone loss (note: any vertical slice through the figure will sum to 100). We note here the dramatic decrease in the escape cone losses with increasing alignment, accompanied by increases in captured photons; the capture rate also significantly exceeds the efficiency losses.

\section{SUMMARY}

We have presented a comprehensive development of a model for photon transport in systems consisting of dye molecules wherein some control over the alignment of the emission and adsorption dipoles is considered. The model is used to perform numerical photon transport simulations of slab geometry solar collector/concentrator devices. The main idea is that control over the alignment of the emission and absorption dipoles can greatly reduce both self-absorption and escape cone losses; a large angle between emission and absorption dipoles reduces the probability for reabsorption, since the emission process polarizes the emitted photon, and if the absorption dipoles are not well-aligned with the emission dipoles, the probability of reabsorption is reduced irrespective of the overlap of the absorption and emission spectral distributions. Furthermore, alignment of emission dipole axes roughly perpendicular to the slab faces fixes the major-

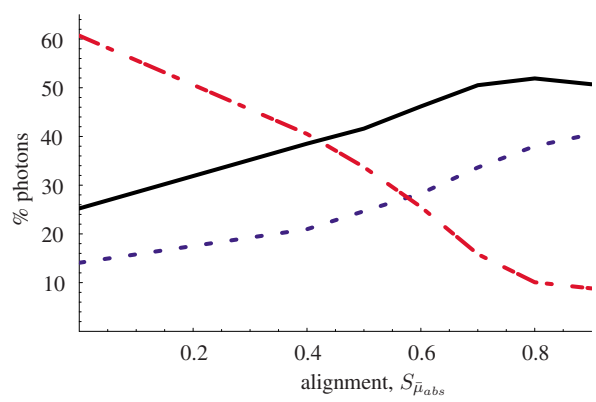

FIG. 10. (Color online) The percentage of photons that are captured (solid line), lost via fluorescent quantum efficiency (dashed line), and escape (dashed-dotted line). ity of photon paths outside of the escape cone-the photon velocities lie in the plane perpendicular to the dipole axis. We have constructed a model that connects knowledge/ control of the molecular alignment in a statistical sense, thus making contact with experimental protocols for dye alignment. The results of our simulations indicate that significant gains in the overall optical quantum efficiency of slab geometry are possible for relatively modest control of alignment, coupled with achievable internal angels between the emission and absorption dipole moments.

\section{ACKNOWLEDGMENTS}

This work was supported by the National Science Foundation, under Grant No. DMR-0705908 and DMS-0553223.

${ }^{1}$ W. H. Weber and J. Lambe, Appl. Opt. 15, 2299 (1976).

${ }^{2}$ A. Goetzberger and W. Greube, Appl. Phys. A: Mater. Sci. Process. 14, 123 (1977).

${ }^{3}$ J. S. Batchelder, A. H. Zewail, and T. Cole, Appl. Opt. 18, 3090 (1979).

${ }^{4}$ J. S. Batchelder, A. H. Zewail, and T. Cole, Appl. Opt. 20, 3733 (1981).

${ }^{5}$ J. Roncali and F. Garnier, Appl. Opt. 23, 2809 (1984).

${ }^{6}$ W. R. L. Thomas, J. M. Drake, and M. L. Lesiecki, Appl. Opt. 22, 3440 (1983).

${ }^{7}$ R. M. Swanson, Prog. Photovolt. Res. Appl. 8, 93 (2000).

${ }^{8}$ W. G. Van Sark, K. W. Barnham, L. H. Slooff, A. J. Chatten, A. Büchtemann, A. Meyer, S. J. McCormack, R. Koole, D. J. Farrell, R. Bose, E. E. Bende, A. R. Burgers, T. Budel, J. Quilitz, M. Kennedy, T. Meyer, S. H. Wadman, G. P. van Klink, G. van Koten, A. Meijerink, and D. Vanmaekelbergh, Opt. Express 16, 21773 (2008).

${ }^{9}$ M. Grell and D. D. C. Bradley, Adv. Mater. (Weinheim, Ger.) 11, 895 (1999).

${ }^{10}$ M. G. Debije, D. J. Broer, and C. W. M. Bastiaansen, Proceedings of the 22nd European Photovoltaic Solar Energy Conference, Milan, Italy, 3 September 2007, p. 87.

${ }^{11}$ P. P. C. Verbunt, A. Kaiser, K. Hermans, C. W. M. Bastiaansen, D. J. Broer, and M. G. Debije, Adv. Funct. Mater. 16, 1 (2009).

${ }^{12}$ M. G. Debije, C. W. M. Bastiaansen, D. J. Broer, and M. J. Escuti Carlos, U.S. Patent Application No. US2009/0027872 (pending).

${ }^{13}$ A. V. Ivashchenko, Dichroic Dyes for Liquid Crystal Displays (CRC, Boca Raton, 1994).

${ }^{14}$ L. R. Dalton, P. A. Sullivan, and D. H. Bale, Chem. Rev. 110, 25 (2010).

${ }^{15}$ G. N. Plass and G. W. Kattawar, Appl. Opt. 7, 415 (1968).

${ }^{16}$ D. Boas, J. Culver, J. Stott, and A. Dunn, Opt. Express 10, 159 (2002).

${ }^{17}$ M. Carrascosa, S. Unamuno, and F. Agullo-Lopez, Appl. Opt. 22, 3236 (1983).

${ }^{18}$ J. Sansregret, J. M. Drake, W. R. Thomas, and M. L. Lesiecki, Appl. Opt. 22, 573 (1983).

${ }^{19}$ M. Bendig, J. Hanika, H. Dammertz, J. C. Goldschmidt, M. Peters, and M. Weber, IEEE Symposium on Interactive Ray Tracing 2008, edited by S. Parker and A. Reshetov (IEEE, New York, 2008), p. 93.

${ }^{20}$ A. R. Burgers, L. H. Slooff, R. Kinderman, and J. A. M. van Roosmalen, Proceedings of the 20th European Photovoltaic Solar Energy Conference, Barcelona, Spain, 6 June 2005, p. 394.

${ }^{21}$ In some experimental arrangements it is possible to determine both $P_{2}\left(\cos \theta_{\bar{\mu}_{\mathrm{em}}}\right)$ and $P_{4}\left(\cos \theta_{\bar{\mu}_{\mathrm{em}}}\right)$, providing even more information on dye orientation. Here, we consider the minimal case where only the former is known. When $P_{4}\left(\cos \theta_{\bar{\mu}_{\text {em }}}\right)$ is known as well, Eqs. (9) and (10) can be modified accordingly to make use of the additional information; See, for example, C. Zanoni in Polarized Spectroscopy of Ordered Systems, edited by B. Samori and E. W. Thulstrop (Kluwer, Dordrecht 1987).

${ }^{22}$ M. J. Currie, J. K. Mapel, T. D. Heidel, S. Goffri, and M. A. Baldo, Science 321, 226 (2008).

${ }^{23}$ J. R. Lakowicz, Principles of Fluorescence Spectroscopy, 3rd ed. (Springer, New York, 2006).

${ }^{24}$ Parameters are for Coumarin 6, see, G. A. Reynolds and K. H. Drexhage, Opt. Commun. 13, 222 (1975).

${ }^{25}$ Parameters are for a mixture of $30 \%$ rubrene/1\% 4-(dicyanomethylene)-2t-butyl-6-(1,1,7,7-tetramethyljulolidyl-9-enyl)-4H-pyran/69\% tris(8hydroxyquinoline) aluminum (AlQ3) with an assumed fluorescent quantum yield $p_{0}=0.90$, see Ref. 22 above. 